\title{
Linked and knotted beams of light
}

\author{
WILLIAM T. M. IRVINE',2* AND DIRK BOUWMEESTER ${ }^{2,3}$ \\ ${ }^{1}$ Center for Soft Condensed Matter Research, Department of Physics, New York University, New York 10003, USA \\ ${ }^{2}$ Department of Physics, University of California, Santa Barbara, California 93106, USA \\ ${ }^{3}$ Huygens Laboratory, Leiden University, PO Box 9504, 2300 RA Leiden, The Netherlands \\ *e-mail: wi1@nyu.edu
}

Maxwell's equations allow for curious solutions characterized by the property that all electric and magnetic field lines are closed loops with any two electric (or magnetic) field lines linked. These little-known solutions, constructed by Rañada ${ }^{1}$, are based on the Hopf fibration. Here we analyse their physical properties to investigate how they can be experimentally realized. We study their time evolution and uncover, through a decomposition into a spectrum of spherical harmonics, a remarkably simple representation. Using this representation, first, a connection is established to the Chandrasekhar-Kendall curl eigenstates ${ }^{2}$, which are of broad importance in plasma physics and fluid dynamics. Second, we show how a new class of knotted beams of light can be derived, and third, we show that approximate knots of light may be generated using tightly focused circularly polarized laser beams. We predict theoretical extensions and potential applications, in fields ranging from fluid dynamics, topological optical solitons and particle trapping to cold atomic gases and plasma confinement.

The concept of field lines whose tangents are the electric or magnetic field is typically used to visualize static solutions of Maxwell's equations. Propagating solutions often have simple field-line structures and so are not usually described in terms of field lines. In the present work, we study a propagating field whose defining and most striking property is the topological structure of its electric and magnetic field lines.

An intriguing configuration for field lines is to be linked and/or knotted. Two closed field lines $\mathbf{c}_{1}(\tau), \mathbf{c}_{2}(\tau)$ are linked if they have non-vanishing Gauss linking integral ${ }^{3-6}$,

$$
L\left(\mathbf{c}_{1}, \mathbf{c}_{2}\right)=\frac{1}{4 \pi} \int \frac{\mathrm{d} \mathbf{c}_{1}}{\mathrm{~d} \tau_{1}} \cdot \frac{\mathbf{c}_{1}-\mathbf{c}_{2}}{\left|\mathbf{c}_{1}-\mathbf{c}_{2}\right|^{3}} \times \frac{\mathrm{d} \mathbf{c}_{2}}{\mathrm{~d} \tau_{2}} \mathrm{~d} \tau_{1} \mathrm{~d} \tau_{2},
$$

whereas for a single field line $\mathbf{c}(\tau)$ the self-linking number, $L(\mathbf{c}, \mathbf{c})$, is a measure of knottedness. The linking integral $L$ can also be computed visually by projecting the field lines onto a plane and subsequently counting the crossings in an oriented way ${ }^{3}$. For example, the lines in Fig. 1a have linking number 1, but do not form a knot, whereas the blue and orange field lines in Fig. 4 below are knotted and linked to each other. In the case of magnetic or electric fields, averaging the linking integral over all field-line pairs together with the self-linking number over all field lines gives rise to the magnetic and electric helicities ${ }^{4,5}$ :

$$
h_{\mathrm{m}}=\int \mathrm{d}^{3} x \mathbf{A}(x) \cdot \mathbf{B}(x) \quad h_{\mathrm{e}}=\int \mathrm{d}^{3} x \mathbf{C}(x) \cdot \mathbf{E}(x),
$$

where $\mathbf{B}:=\nabla \times \mathbf{A}$ and $\mathbf{E}:=\nabla \times \mathbf{C}$ in free space.

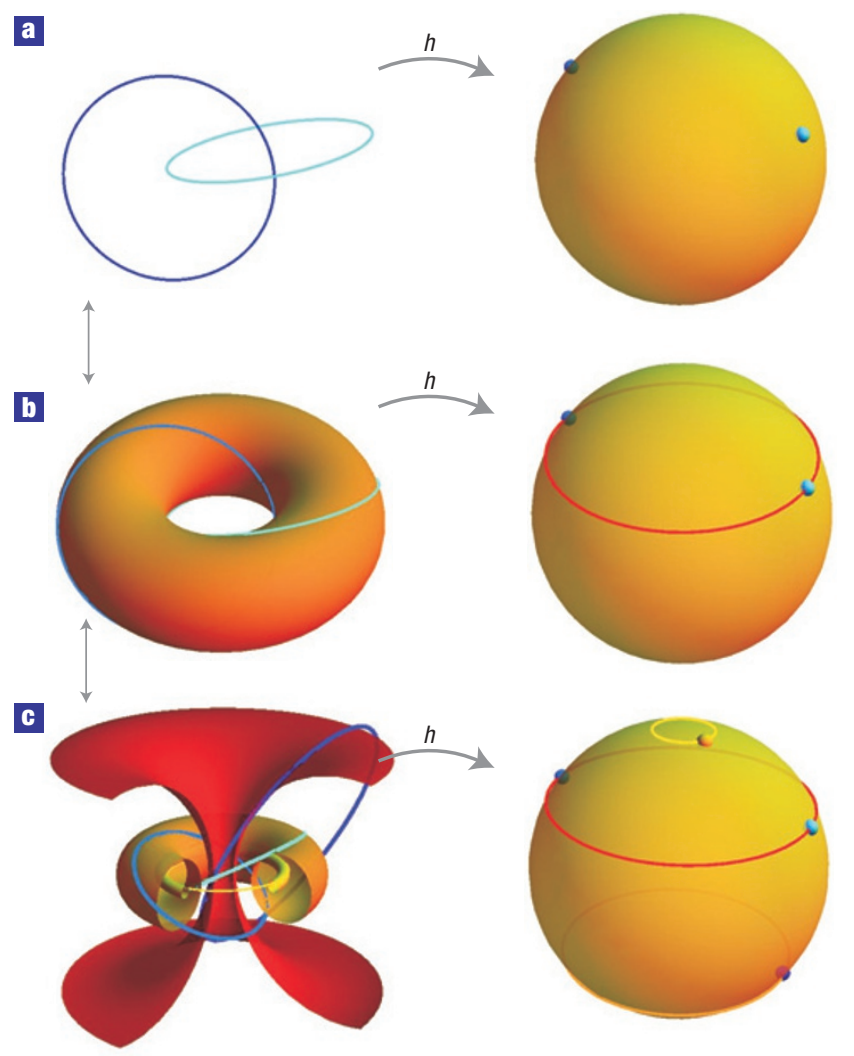

Figure 1 Construction of the Hopf fibration. a-c, Left column: A torus can be constructed out of circles (fibres) in such a way that no two circles cross and each circle is linked to every other one. $\mathbf{a}, \mathbf{b}$, Each circle in such a configuration wraps once around each circumference of the torus. c, By nesting such tori into one another, the whole of three dimensional space, including the point at $r=\infty$ $\left(\mathbb{R}^{3} \cup \infty \sim \mathbb{S}^{3}\right)$ can be filled with linked circles. There are two 'special' fibres: the circle of unit radius that corresponds to the infinitely thin torus, and the straight line, or circle of infinite radius, that corresponds to an infinitely large torus. These two fibres will provide an economical way of characterizing the time evolution of the configuration. Right column: The Hopf map maps such circles in $\mathbb{R}^{3} \cup \infty \sim \mathbb{S}^{3}$ to points on the sphere $\mathbb{S}^{2} \sim C \cup \infty$. Each circle is mapped to a point, each torus in $\mathbb{R}^{3} \cup \infty$ onto a (parallel) circle on $\mathbb{S}^{2}$. The circular (straight) special fibres are mapped to the north (south) pole and will be referred to as the $n(s)$ fibres. In the present work, the fibres of two everywhere-orthogonal Hopf fibrations correspond to electric and magnetic field lines (see Fig. 2 for $t=0$ ). 
Since Kelvin proposed knotted field configurations as a model for atoms, knots and links have been studied in branches of physics as diverse as fluid dynamics ${ }^{7}$, plasma ${ }^{5}$ and polymer physics ${ }^{6}$. More recently, an approach to knotted classical fields was proposed ${ }^{8}$ and further understood and developed ${ }^{9,10}$. Knotted vortex lines have also been considered in phases associated with the electron states of hydrogen ${ }^{11}$, with the Riemann-Silberstein vector of the electromagnetic field ${ }^{12}$ and in phases associated with lines of darkness in a monochromatic light field ${ }^{13}$, with the latter predictions experimentally verified ${ }^{14}$.

Here we consider a state of light whose electric field lines are all closed and any two are linked to each other as described in Figs 1 and 2. The magnetic and Poynting field lines are similarly arranged. This structure is based on the Hopf fibration defined by the Hopf map $h: \mathbb{S}^{3} \rightarrow \mathbb{S}^{2}$ (see Fig. 1) ${ }^{15,16}$. Using stereographic projections, $h$ can in turn be expressed as a complex function in $\mathbb{R}^{3}$ (for example $\zeta(x, y, z, 0)$ or $\eta(x, y, z, 0)$ below) whose lines of constant amplitude and phase are circles, and surfaces of constant amplitude are nested tori.

Electromagnetic fields derived from the Hopf fibration first appeared in ref. 17, and were extended to propagating solutions by Rañada in refs $1,18,19$. The construction was cast in terms of differential forms, which provide a natural way to map fields between spaces of differing dimensions. The resulting electric and magnetic fields have simple expressions:

$$
\begin{aligned}
& \mathbf{B}=\frac{1}{4 \pi i} \frac{\nabla \eta \times \nabla \bar{\eta}}{(1+\bar{\eta} \eta)^{2}} ; \quad \mathbf{E}=\frac{1}{4 \pi i} \frac{\nabla \zeta \times \nabla \bar{\zeta}}{(1+\bar{\zeta} \zeta)^{2}}, \\
& \zeta(x, y, z, t)=\frac{(A x+t y)+i(A z+t(A-1))}{(t x-A y)+i(A(A-1)-t z)}, \\
& \eta(x, y, z, t)=\frac{(A z+t(A-1))+i(t x-A y)}{(A x+t y)+i(A(A-1)-t z)},
\end{aligned}
$$

where $A=\frac{1}{2}\left(x^{2}+y^{2}+z^{2}-t^{2}+1\right)$, and $x, y, z, t$ are dimensionless multiples of a length scale $a$. Since both $\nabla \eta$ and $\nabla \bar{\eta}$ are perpendicular to lines of constant $\eta$, the magnetic field is tangential to lines of constant $\eta$. A similar argument holds for the electric field and $\zeta$. The corresponding field lines are shown in Fig. 2.

As a first step in our investigation, we present a numerical study of the evolution of the field lines and energy density, shown in Fig. 2. The initially spherical energy density expands like an 'opening umbrella' with a preferred propagation direction $(z)$ while preserving the Hopf structure. The propagation direction is set by the cross-product of the electric and magnetic $n$ lines (as defined in Fig. 1). The $s$ fibre twists around the centre of energy density. The $n$ fibre cuts through the maximum of energy density and its tangent on the $z$ axis undergoes a rotation with an angle analogous to the Gouy phase shift of Gaussian beam optics ${ }^{20}$.

To further characterize the physical properties of the field configuration, we compute the full set of conserved quantities that correspond to the known (conformal) symmetries of electromagnetism in free space (see Table 1). Note that all currents, when scaled by the energy density and the scale factor $a$, are integer multiples of one another; that the fields carry angular momentum along the propagation axis and that the momentum is a fraction of the energy, so the Hopf fields can be transformed via a Lorentz transformation to a rest frame, or to a counter-propagating frame, making them even more beamlike.

Though the linking number is also a conserved quantity for the solution under consideration, it does not correspond to a
Table 1 Conserved (Noether) currents and charges of free-space electromagnetism and their value for the knots considered here. The centre column gives expressions for the energy density (corresponding to time-translation invariance), momentum density (space translations), angular momentum density (rotations), boost vector density (Lorentz boosts), special conformal current and charge densities (special conformal transformations, SCT) and dilation charge density (scale invariance). Expressions for the more familiar non-SCT currents can be found in most classical field-theory texts. The special conformal current and charge were computed following the Noether procedure for the special conformal transformations ${ }^{33}$. The rightmost column gives the values of the currents and charges carried by the knots obtained by integrating the current densities over all space. The length scale $a=1$ and the values are rescaled by the energy density.

\begin{tabular}{lll}
\hline Charge & Density & Value \\
\hline Energy $(E)$ & $\frac{1}{2}(\mathbf{E} \cdot \mathbf{E}+\mathbf{B} \cdot \mathbf{B})$ & 1 \\
Momentum $(\mathbf{P})$ & $\mathbf{E} \times \mathbf{B}$ & $\left(0,0, \frac{1}{2}\right)$ \\
Ang. mom. $(\mathbf{L})$ & $(\mathbf{E} \times \mathbf{B}) \times \mathbf{x}$ & $\left(0,0,-\frac{1}{2}\right)$ \\
Boost vector & $E \mathbf{x}-\mathbf{P} t$ & $(0,0,0)$ \\
SCT (q) & $\left(\mathbf{x}^{2}+t^{2}\right) E+t \mathbf{P} \cdot \mathbf{x}$ & 1 \\
SCT $(\mathbf{v})$ & $2 \mathbf{x}(\mathbf{P} \cdot \mathbf{x})-2 t E \mathbf{x}-\mathbf{x}^{2} \mathbf{P}$ & $\left(0,0, \frac{1}{2}\right)$ \\
Dilation & $\mathbf{P} \cdot \mathbf{x}-E t$ & 0 \\
\hline
\end{tabular}

space-time symmetry; rather, it is a topological invariant ${ }^{4}$. Indeed, the linking number is not conserved for a general free-space electromagnetic field, but only for fields that satisfy

$$
\partial_{t} \int \mathbf{A} \cdot \mathbf{B} \propto \int \mathbf{E} \cdot \mathbf{B}=0 .
$$

In the case of the Hopf fields defined in equation (2), $\mathbf{E} \cdot \mathbf{B}=0$ guarantees the conservation of linking number.

For problems with spherical symmetry, a natural basis for representing electromagnetic fields is that of the vector spherical harmonics (VSPHs). Labelled by angular-momentum integers $l \geq 1$ and $-l \leq m \leq l$, wavevector $k$ and polarization TE/TM (electric/magnetic field transverse to the radial direction), the vector potential $\mathbf{A}_{l m}(k, \mathbf{r})$ for the VSPHs is ${ }^{21}$

$$
\begin{gathered}
\mathbf{A}_{l m}^{\mathrm{TE}}(k, \mathbf{r})=\frac{1}{i \omega} f_{l}(k r) \mathbf{L} Y_{l m}(\theta, \phi), \\
\mathbf{A}_{l m}^{\mathrm{TM}}(k, \mathbf{r})=\frac{1}{k^{2}} \nabla \times\left[f_{l}(k r) \mathbf{L} Y_{l m}(\theta, \phi)\right],
\end{gathered}
$$

where $\mathbf{L}=-i \mathbf{r} \times \nabla, Y_{l m}$ are spherical harmonics and $f_{l}(k r)$ is a linear combination of the spherical Bessel functions $j_{l}(k r)$, $n_{l}(k r)$, determined by boundary conditions. In free space $f_{l}(k r)=j_{l}(k r) / \sqrt{l(l+1)}$. A general free-space vector potential $\mathbf{A}(\mathbf{r}, t)$ can be expressed in the spherical harmonic basis as

$$
\begin{aligned}
\mathbf{A}(\mathbf{r}, t)= & \int \mathrm{d} k \sum_{l=1}^{\infty} \sum_{m=-l}^{l}\left[\alpha_{l m}^{\mathrm{TM}}(k) \mathbf{A}_{l m}^{\mathrm{TM}}(k, \mathbf{r})\right. \\
& \left.+\alpha_{l m}^{\mathrm{TE}}(k) \mathbf{A}_{l m}^{\mathrm{TE}}(k, \mathbf{r})\right] \mathrm{e}^{-i \omega t}+\text { c.c. }
\end{aligned}
$$

We present the decomposition of the Hopf electromagnetic field in this basis (see Supplementary Information, Methods S1), revealing 

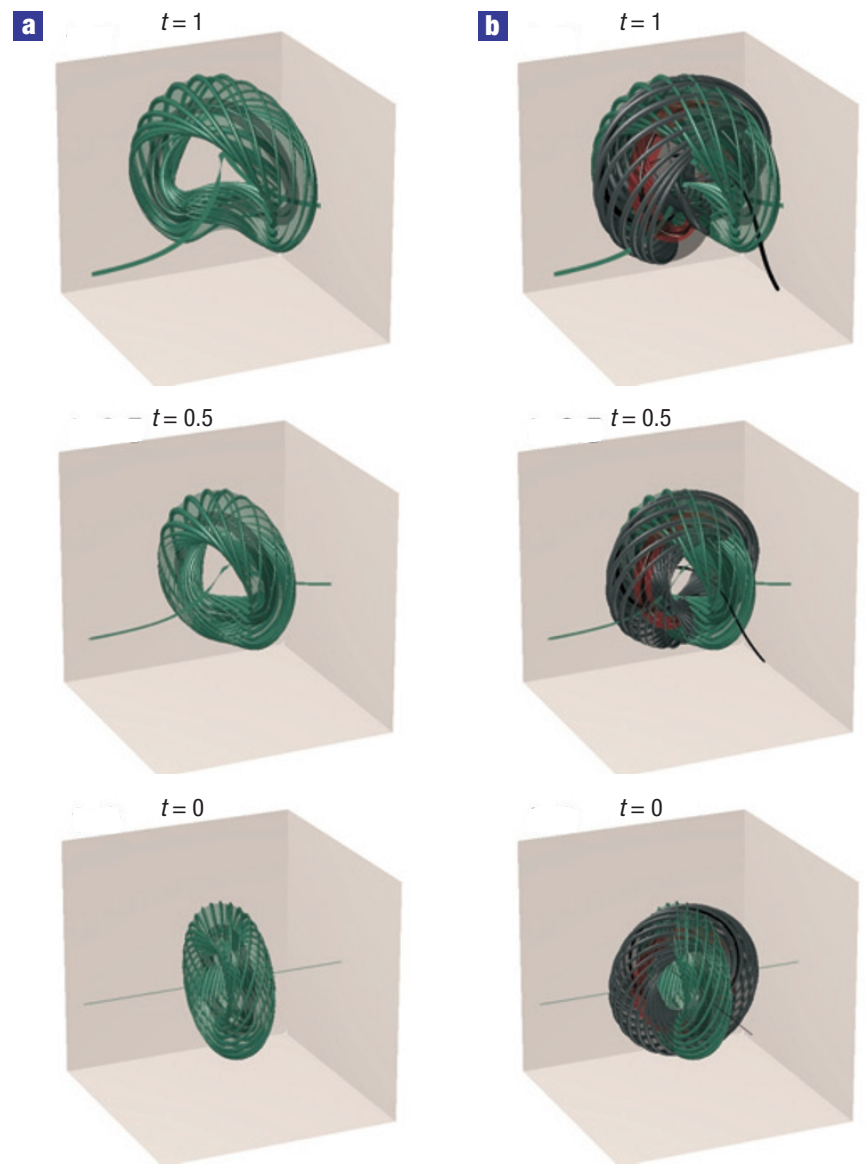

G
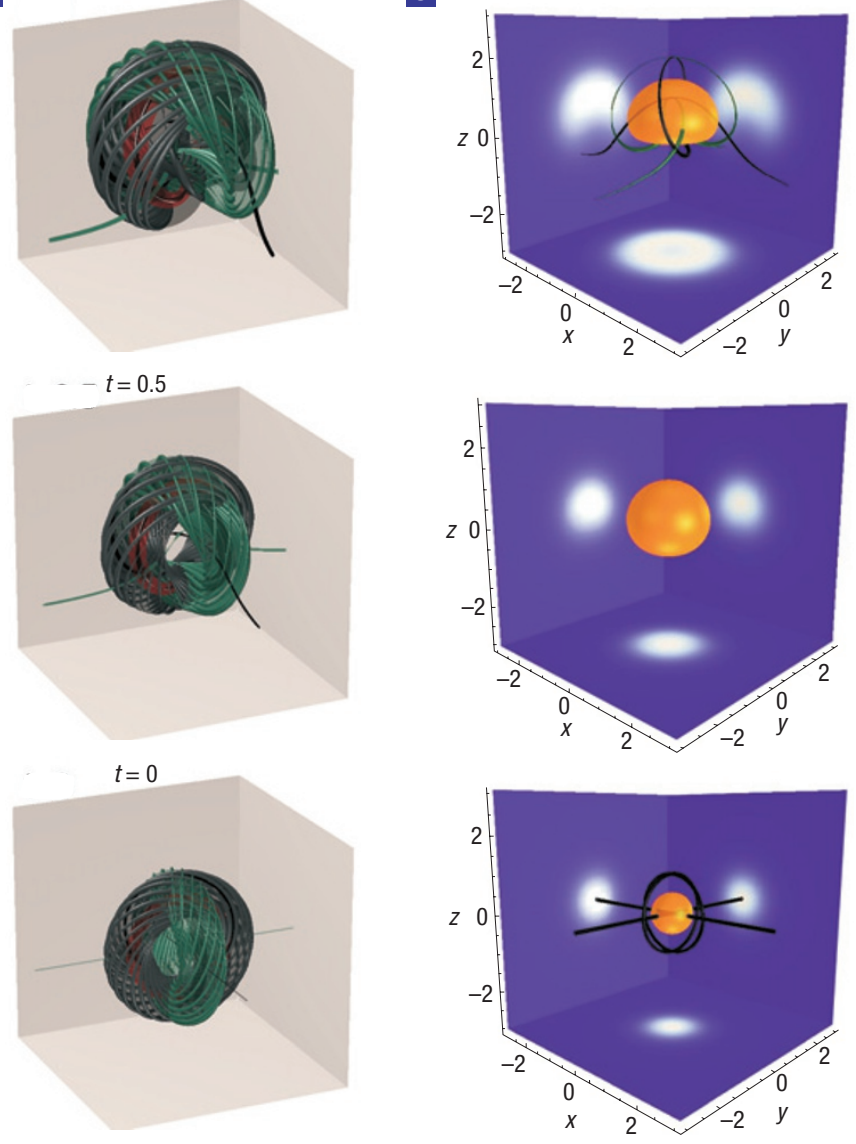

Figure 2 Time evolution of the field lines and energy density of the Hopf knot. a,b, Time evolution of the electric field lines (green) (a) and of the magnetic field lines (black) shown together with the electric field lines for reference (b; see also Supplementary Information, Videos S1-S3). At time $t=0$, the Hopf fibration discussed in Fig. 1 can be clearly recognized with the $n$ fibres aligned with the $x$ (electric-field) and $y$ (magnetic-field) axes, and the $s$ fibres lying in the $y-z$ (electric-field) and $x-z$ (magnetic-field) planes, centred on the origin. The same sets of fibres are shown at times $t=0.5$ and 1 . c, Time evolution of the energy density, which is initially spherical and then propagates and expands like an 'opening umbrella' along the $z$ axis. The fibration can be seen to locally rotate about the $z$ axis as well as expanding and deforming, with the structure remaining centred on the centre of energy of the knot. The rotation and deformation seen at these times slows down dramatically at subsequent times (see Fig. 4e), in an analogous way to the variation of the Gouy phase of a focused Gaussian beam ${ }^{20}$. The electric and magnetic field lines remain perpendicular everywhere throughout the time evolution.

the following remarkably simple structure:

$$
\begin{aligned}
\mathbf{A}_{\text {Hopf }}(\mathbf{r}, t)= & \sqrt{\frac{4}{3 \pi}} \int \mathrm{d} k k^{3} \mathrm{e}^{-k}\left[\mathbf{A}_{1,1}^{\mathrm{TE}}(k, \mathbf{r})\right. \\
& \left.-i \mathbf{A}_{1,1}^{\mathrm{TM}}(k, \mathbf{r})\right] \mathrm{e}^{-i \omega t}+\text { c.c.; }
\end{aligned}
$$

the Hopf field is a superposition of TE and TM vector spherical harmonics corresponding to a single multipole $(l=m=1)$, a relative phase factor $i$ and an energy spectrum

$$
S(\omega) \sim \omega \mathrm{e}^{-\omega}
$$

Strikingly, the superposition $\mathbf{A}^{\mathrm{TE}}-i \mathbf{A}^{\mathrm{TM}}$ is an eigenstate of the curl operator, that is, it satisfies the eigenvalue equation

$$
\boldsymbol{\nabla} \times\left[\mathbf{A}_{l m}^{\mathrm{TE}}(k) \pm i \mathbf{A}_{l m}^{\mathrm{TM}}(k)\right]=\mp k\left[\mathbf{A}_{l m}^{\mathrm{TE}}(k) \pm i \mathbf{A}_{l m}^{\mathrm{TM}}(k)\right]
$$

Such eigenstates, known as Chandrasekhar-Kendall $(\mathrm{CK})^{2}$ states for constant $k$, are part of a family of fields known as force-free fields and are of broad importance in plasma physics and fluid dynamics $^{22-24}$. The Hopf fields are therefore a pulsed version of the CK curl eigenstate fields with energy spectrum $\omega \mathrm{e}^{-\omega}$.

To understand how such a simple superposition (equation (4)) gives rise to the remarkable field-line structure of the Hopf field, we begin by studying, in Fig. 3, the field lines of the single-frequency curl eigenstates, which have the unique property that the electric, magnetic and A-field lines have the same structure up to a rotation $(\mathbf{B}=\nabla \times \mathbf{A}= \pm k \mathbf{A}, \mathbf{E}=-\dot{\mathbf{A}}=i \omega \mathbf{A})$. The $\mathbf{A}_{1,1}^{\mathrm{TE}}$ and $i \mathbf{A}_{1,1}^{\mathrm{TM}}$ field lines are symmetric under rotations about the centre axis and separate into sets of nested tori, with each set centred on zeros of the field. The $\mathbf{A}_{1,1}^{\mathrm{TE}}$ field lines follow one and the $i \mathbf{A}_{1,1}^{\mathrm{TM}}$ lines the other circumference of each torus. A superposition will therefore have field lines that stay confined and wrap around the tori with linking (winding ratio) that depends on the field strength (Fig. 3b).

To understand the step from the single-frequency curl eigenstates to a Hopf configuration, it is necessary to take the energy spectrum into consideration. To nest all tori about the same $s$ 
a

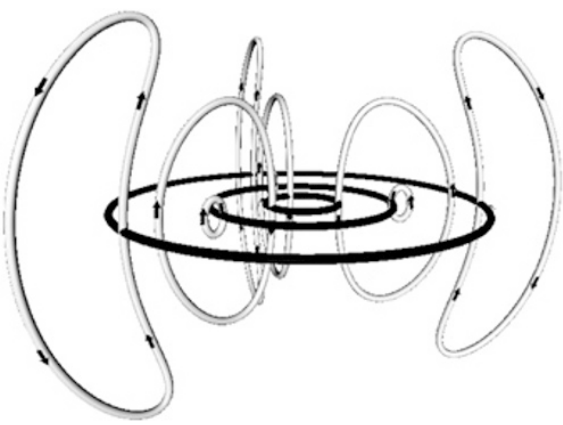

b
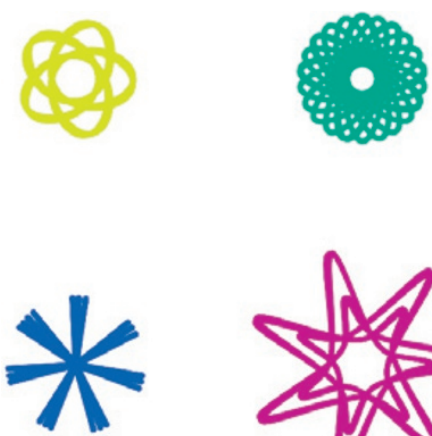

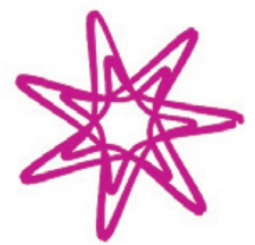

Figure 3 Field-line structure of single-frequency CK curl eigenstates. For such states, the electric, magnetic and A-field lines have similar structure. a, Field lines of $\mathbf{A}_{1,1}^{\mathrm{TE}}$ (white) and $i \mathbf{A}_{1,1}^{\mathrm{TM}}$ (black) spherical harmonic fields at time $t=0$. Both sets of field lines are symmetric under rotations about the centre axis. The $\mathbf{A}_{1,1}^{\mathrm{TE}}$ field lines make up a collection of sets of nested deformed tori with the field lines following one of the torus circumferences. The $i \mathbf{A}_{1,1}^{\mathrm{TM}}$ are circles centred on and perpendicular to the centre axis and therefore follow the other circumference of the nested tori. The field-line direction is reversed in the centre of each set, corresponding to zeros in the field strength. A superposition of $\mathbf{A}_{1,1}^{\mathrm{TE}}$ and $i \mathbf{A}_{1,1}^{\mathrm{TM}}$ will therefore have field lines that wrap around the tori and stay confined to them; the ratio of the winding around each circumference will vary depending on the strength of the field at these points. $\mathbf{b}$, Some of the resulting field lines on different tori, with different winding ratios.

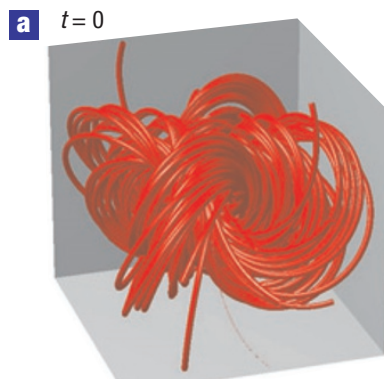

d

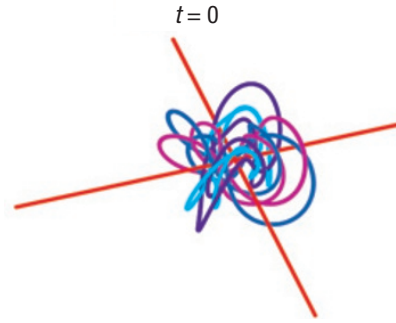

e

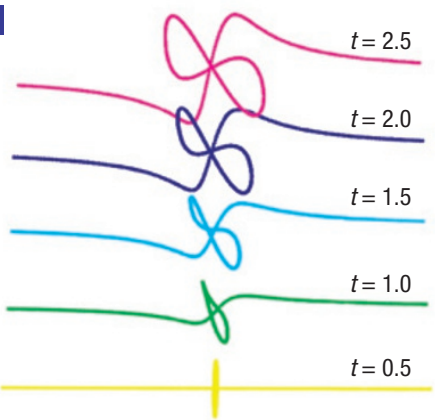

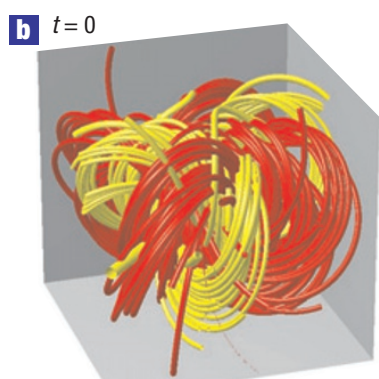
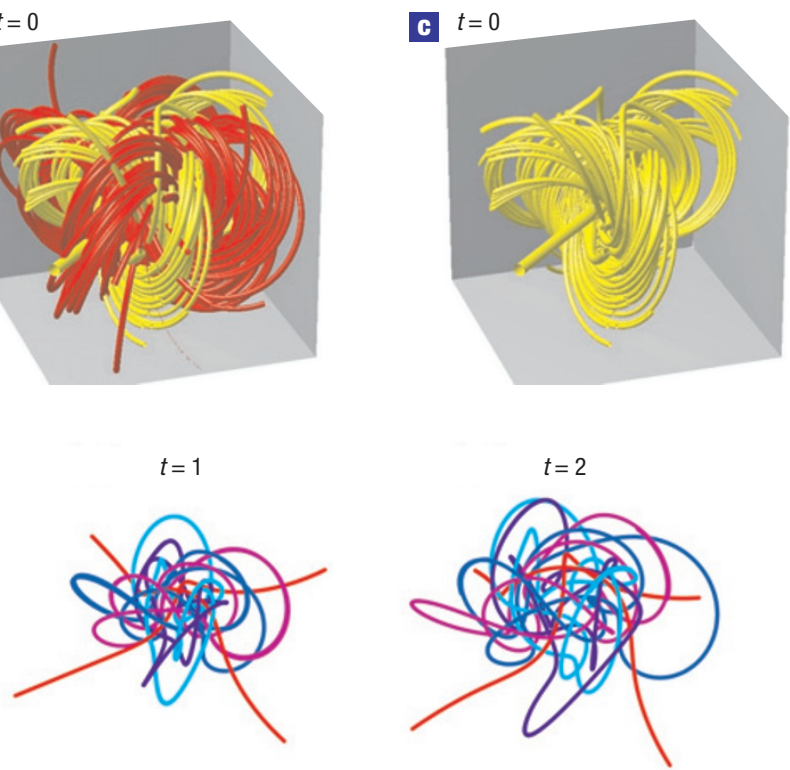

$\mathbf{f}$
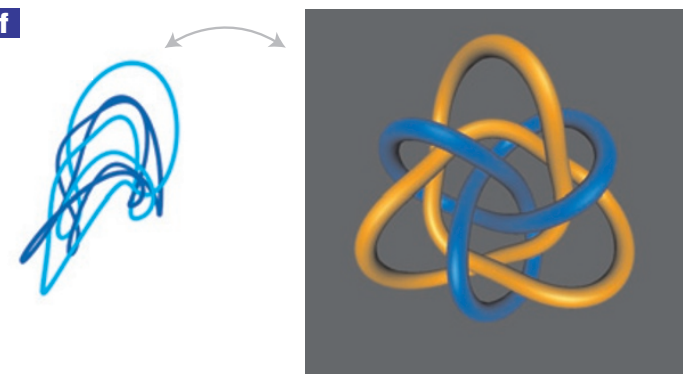

Figure 4 A generalization of the Hopf fields on the basis of the VSPHs, obtained using $I=2, m=2$ multipole fields and the spectrum of equation (5). a-c, The magnetic (red) and electric (yellow) field lines at time $t=0$. d, within these field lines we have numerically found a set of fibres that are analogous to the $s$ and $n$ fibres of the Hopf fields in that their structure is preserved by time evolution. There are two (red) lines analogous to the $s$ fibre and four analogous to the $n$ fibre. e, The time evolution of the $s$ and $n$ fibres for the Hopf field for comparison. $\mathbf{f}$, The structure of the fibres analogous to the $n$ fibre can be better understood by picking a pair and allowing it to 'relax' using a package for representing knots ${ }^{32}$, revealing doubly linked trefoil knots. 
fibre, the spectrum must 'eliminate' all zeros of the radial function, effectively giving rise to a field without oscillations. This is achieved by the spectrum in equation (5). Interestingly, this spectrum is close to one used in research on single-cycle light 'bullets' ${ }^{25}$ based on the fields of Ziolkowski ${ }^{26}$, whose defining property is the absence of oscillations.

We now consider how the linking is preserved in time. A curl eigenstate has conserved helicity because its helicity integral is proportional to the norm of the state. A calculation of the helicity integral (equation (1)) for a general field in the CK basis gives

$$
\begin{aligned}
\mathcal{L} \propto & \int \frac{\mathrm{d} k}{k^{3}}\left[\left|\alpha_{l m}^{+}(k)\right|^{2}-\left|\alpha_{l m}^{-}(k)\right|^{2}\right] \\
& -\frac{1}{2}\left[\alpha_{l m}^{+}(k) \alpha_{l,-m}^{+}(k)-\alpha_{l m}^{-}(k) \alpha_{l,-m}^{-}(k)\right] \mathrm{e}^{-i 2 \omega t}+\text { c.c. },
\end{aligned}
$$

where $\alpha^{ \pm}=\frac{1}{\sqrt{2}}\left(\alpha^{\mathrm{TE}} \pm i \alpha^{\mathrm{TM}}\right)$ of equation (3) are the coefficients of the CK eigenstates. Only the second term is time dependent; the absence of $((l, m),(l,-m))$ pairs therefore guarantees the conservation of helicity. The conserved part (first term) of the linking number is proportional to the difference between the amplitudes of the + and - CK states.

The understanding gained above suggests a route to generalizing these solutions: taking superpositions of curl states with different values of $l$ and $m$ and a similar energy spectrum. The result for $l=2, m=2$, is shown in Fig. 4 . Not all the field lines are closed and link in the same way, as is the case for the Hopf fields; however, fibres analogous to the $s$ and $p$ fibres of the Hopf knot can be found. These fibres close, follow the energy density and have a fixed linking structure in the form of two intertwined trefoil knots. In addition, we note that by varying the relative strength of the $\mathrm{TE}$ and TM components in the single-frequency building blocks, $p \mathbf{A}^{\mathrm{TE}}+i q \mathbf{A}^{\mathrm{TM}}$, all possible torus knots with winding $p, q$ can be produced at time $t=0$.

We now turn to the possibility of an experimental realization using laser fields. A simple argument suggests that the relative phase factor of the CK building blocks may be fairly robust: pure TE and TM free-space VSPHs are a simple superposition of out-going and in-going VSPHs; their time-averaged Poynting vector is indeed purely azimuthal. The only way to construct a pure multipole field that propagates along the $z$ axis in free space is by taking a superposition of TE and TM fields with a phase $i$; therefore, any propagating pure multipole is a Chandrasekhar-Kendall curl eigenstate.

The fact that the Hopf fields are built of CK states with only one value of $l$ and $m$ suggests Laguerre-Gaussian beams ${ }^{20}$ as a good starting point for their production; these are pure angularmomentum eigenstates of the paraxial wave equation and are used as a basis to model laser beams. Though studied extensively, only recently has the relation between strongly focused laser beams and VSPHs received some attention ${ }^{27,28}$, motivated in part by optical tweezing. Using the code developed in ref. 28 provided to us by the authors, we found that a strongly focused zeroth-order Gaussian beam with circular polarization converges towards a pure $l=1, m=1$ multipole field as the focusing angle increases toward $90^{\circ}$. This suggests that an experimental implementation of the CK basis states may be simpler than we might expect. To create the full Hopf field we would start with a single- or few-cycle pulsed beam of circularly polarized light ${ }^{29}$ and focus it tightly. The pulse shape and spatial profile could be further controlled with a spatial light modulator using holographic techniques ${ }^{30}$, which have been recently used to produce, for example, pure Airy beams ${ }^{31}$.
In conclusion, we have investigated the physical properties of an exceptional solution of the charge-free Maxwell equations in which all field lines are linked once with one another. The decomposition into vector spherical harmonics has revealed the relation to eigenstates of the curl operator, led the way to new field configurations with multiple linking and given guidance on how to generate such special solutions in an experiment. Since the class of electromagnetic knots has both beamlike propagation and unique properties that have not been explored in this context, we predict a wide variety of potential applications and theoretical extensions in areas ranging from colloidal and atomic particle trapping to manipulating cold atomic ensembles and from generating soliton-like solutions in nonlinear media to helicity injection for plasma confinement.

Received 17 March 2008; accepted 24 July 2008; published 31 August 2008.

\section{References}

1. Rañada, A. F. A topological theory of the electromagnetic field. Lett. Math. Phys. 18, 97-106 (1989). 2. Chandrasekhar, S. \& Kendall, P. C. On force-free magnetic fields. Astrophys. J. 126, 457-460 (1957).

3. Rolfsen, D. Knots and Links (Publish or Perish, California, 1976).

4. Baez, J. C. \& Muniain, J. P. Gauge Fields, Knots and Quantum Gravity (World Scientific, Singapore, 1994).

5. Berger, M. A. Introduction to magnetic helicity. Plasma Phys. Control. Fusion 41, B167-B175 (1999).

6. Kamien, R. D. The geometry of soft materials: A primer. Rev. Mod. Phys. 74, 953-971 (2002).

7. Moffatt, H. K. The degree of knottedness of tangled vortex lines. J. Fluid Mech. 35, 117-129 (1969).

8. Faddeev, L. \& Niemi, A. J. Stable knot-like structures in classical field theory. Nature 387, 58-61 (1997).

9. Battye, R. A. \& Sutcliffe, P. M. Knots as stable soliton solutions in a three-dimensional classical field theory. Phys. Rev. Lett. 81, 4798-4801 (1998).

10. Babaev, E., Faddeev, L. D. \& Niemi, A. J. Hidden symmetry and knot solitons in a charged two-condensate bose system. Phys. Rev. B 65, 100512(R) (2002).

11. Berry, M. V. Knotted zeros in the quantum states of hydrogen. Found. Phys. 31, $659-667$ (2001).

12. Bialynicki-Birula, I. \& Bialynicki-Birula, Z. Vortex lines of the electromagnetic field. Phys. Rev. A 67, 062114 (2003).

13. Berry, M. V. \& Dennis, M. R. Knotted and linked phase singularities in monochromatic waves. Proc R. Soc. Lond. A 457, 2251-2263 (2001).

14. Leach, J., Dennis, M. R., Courtial, J. \& Padgett, M. Knotted threads of darkness. Nature 432, 165 (2004).

15. Ward, R. S. \& Wells, R. O. Twistor Geometry and Field Theory (Cambridge Univ. Press, Cambridge, 1990).

16. Urbantke, H. K. The Hopf fibration—seven times in physics. J. Geom. Phys. 46, 125-150 (2003).

17. Trautman, A. Solutions of the Maxwell and Yang-Mills equations associated with Hopf fibrings. Int. J. Theor. Phys. 16, 561-565 (1977).

18. Rañada, A. F. Knotted solutions of the Maxwell equations in vacuum. J. Phys. A 23, L815-L820 (1990).

19. Rañada, A. F. \& Trueba, J. L. Topological electromagnetism with hidden nonlinearity. Mod. Nonlin. Opt. 119, 197-253 (2001).

20. Allen, L., Beijersbergen, M. W., Spreeuw, R. J. C. \& Woerdman, J. P. Orbital angular momentum of light and the transformation of Laguerre-Gaussian laser modes. Phys. Rev. A 45, 8185-8189 (1992).

21. Jackson, J. D. Classical Electrodynamics (Wiley, New Jersey, 1999).

22. Moses, H. E. Eigenfunctions of the curl operator, rotationally invariant Helmholtz theorem, and applications to electromagnetic theory and fluid mechanics. SIAM J. Appl. Math. 21, 114-144 (1971).

23. Rañada, A. F. \& Trueba, J. L. Ball lightning, an electromagnetic knot? Nature 383, 32 (1996).

24. Woltjer, L. A theorem on force free magnetic fields. Proc. Natl Acad. Sci. 44, 489-491 (1958).

25. Feng, S., Winful, H. G. \& Hellwarth, R. W. Spatiotemporal evolution of focussed single-cycle electromagnetic pulses. Phys. Rev. E 59, 4630-4649 (1999).

26. Ziolkowski, R. W. Exact solutions of the wave equation with complex source locations. J. Math. Phys. 26, 861-3 (1985).

27. Nieminen, T. A., Rubinsztein-Dunlop, H. \& Heckenberg, N. R. Multipole expansion of strongly focussed laser beams. J. Quant. Spectrosc. Radiat. Transfer 863, 1005-1017 (2003).

28. Nieminen, T. A. et al. Optical tweezers computational toolbox. J. Opt. A 9, S196-S203 (2007).

29. Apolonski, A. et al. Controlling the phase evolution of few-cycle light pulses. Phys. Rev. Lett. 85, 740-743 (2000).

30. Grier, D. A revolution in optical manipulation. Nature 424, 810-816 (2003).

31. Siviloglou, G. A., Broky, J., Dogariu, A. \& Christodoulides, D. N. Observation of accelerating Airy beams. Phys. Rev. Lett. 99, 213901 (2007).

32. Scharein, R. G. Interactive Topological Drawing. Thesis, Department of Computer Science, The Univ. British Columbia, (1998).

33. Di Francesco, P., Mathieu, P. \& Sénéchal, D. Conformal Field Theory (Springer, New York, 1997).

Supplementary Information accompanies the paper at www.nature.com/naturephysics.

\section{Acknowledgements}

We gratefully acknowledge discussions with M. Srednicki, J. Hartle and K. Millett. We thank V. Vitelli, C. Simon and F. Azhar for comments on the manuscript. W.T.M.I. gratefully acknowledges support from the English Speaking Union through a Lindemann Fellowship. D.B. acknowledges support from Marie Curie EXT-CT-2006-042580.

\section{Author information}

Reprints and permission information is available online at http://npg.nature.com/reprintsandpermissions. Correspondence and requests for materials should be addressed to W.T.M.I. 